\section{Safety of Blunt Spinal Injury Patient on Hospital Gurney}

\author{
WEI-CHE LEE • LIANG-CHI KUO • YUAN-CHIA CHENG • \\ CHAO-WEN CHEN • YEN-KO LIN • TSUNG-YING LIN • HSING-LIN LIN
}

HSING-LIN LIN $(\bowtie)$ WEI-CHE LEE • LIANG-CHI KUO • YUAN-CHIA CHENG • CHAO-WEN CHEN • YEN-KO LIN •

TSUNG-YING LIN

Division of Trauma, Department of Surgery Kaohsiung Medical University, Kaohsiung

Department of Trauma Kaohsiung Medical University Hospital

100 Tzyou 1st Road

Kaohsiung 807, Taiwan

Phone: + 88673125895 ext 7553

Fax: +88673208255

E-mail: hsinglin2002@yahoo.com.tw

WEI-CHE LEE •

LIANG-CHI KUO •

YUAN-CHIA CHENG •

CHAO-WEN CHEN •

YEN-KO LIN •

TSUNG-YING LIN •

HSING-LIN LIN

Department of Emergency Medicine

Kaohsiung Medical University Hospital

Kaohsiung Medical University, Kaohsiung

Taiwan

HSING-LIN LIN

Graduate Institute of Medicine, College of Medicine, Kaohsiung Medical University Kaohsiung, Taiwan

Wei-Che Lee, Hsing-Lin Lin

Department of Emergency Medicine, Faculty

of Medicine, College of Medicine, Kaohsiung Medical University, Kaohsiung, Taiwan

\footnotetext{
ABSTRACT

Objectives. Restraint of patients on a spine board has been used in the past to prevent further spinal cord injury after rescue of patients from the scene of an accident. Removal from the spine board is a routine protocol in many hospitals once the patient has been cleared of spinal injury. However, the benefit of using a spine board, in light-weight motorcycle-related accident victims, has never been studied before.

Materials and methods. A retrospective observational study enrolled patients who had sustained motorcycle-related accidents and were brought to our emergency department (ED). Patients with a Glasgow Coma Scale score lower than 15 and those who died at the ED, or had incomplete data, were excluded. The diagnosis of spinal injury was based upon clinical evaluation and was confirmed by computed tomography, magnetic resonance imaging or X-ray reports, as interpreted by a qualified radiologist. A neurological examination was performed, according to the Standard Neurological Classification of Spinal Cord Injury, directly after arrival and again before leaving the ED.

Results. During the study period, from January 2007 to December 2010, 91 patients with spinal injuries who met the inclusion criteria, consisting of 35 male and 56 female patients with a mean age of $45.44 \pm 18.12$ years, were enrolled in our study. The scores of the motor and sensory neurological exams did not show any significant change during the ED stay after being placed in a gurney without a spine board $(p=0.432)$.

Conclusions. Removal of the spine board and placement on a hospital gurney sponge is safe in alert patients whose primary examination is completed at the ED.
} 
Key words: motorcycle accident, spinal injury, spine board, emergency department

\section{Introduction}

Spinal cord injury is an important issue in trauma patients and immobilization is essential for most patients after suffering a traffic accident. Therefore, most of the victims in traffic accidents brought to hospitals by emergency medical technicians (EMTs), who are trained to immobilize patients as suggested by Advanced Trauma Life Support (ATLS), are placed on an extrication board or given a neck collar out of fear of causing further spinal injuries. (1-3) However, the proper time for removal of the extrication board and the efficacy of immobilization with an extrication board, as compared to those without a stabilization device, has rarely been studied, especially after patients have arrived at the hospital.

According to ATLS guidelines, the extrication board should be removed as soon as spinal injury has been excluded, to prevent discomfort from the hard surface and the possibility that it may cause bedsores or increased occipital and lumbosacral pain in patients that have been lying down for a long period of time. $(4,5)$ There is substantial literature highlighting the problems associated with the extrication board, and this device is now widely accepted as being inappropriate for the transport or care of patients due to these problems. Although it is questionable whether or not all patients rescued from the scene of an accident should be placed on an extrication board with a neck collar, especially in those patients without any signs of spinal injury, a rigid board does not conform to the shape of a patient's back, and has been shown to provide support that is inferior to other methods of immobilization, such as a vacuum or other mattress. Nevertheless, the suitability of applying an extrication board at the scene of an accident, and the time at which the patient should be removed from it in the hospital remain controversial. (6-8)

In this study, we evaluated the patients who were brought to our hospital after motorcycle accidents. All patients were then put in a hospital gurney with a 10 $\mathrm{cm}$ sponge after arrival at the emergency department (ED). These patients were further evaluated for spinal injury and outcome after removal of the board. A neurological examination was performed before and after removal of the extrication board.

\section{Materials and methods}

Study Setting and Sample

The hospital used in this study is a 1,200 bed medical center that provides the service of a tertiary trauma center in Southern Taiwan and serves as a regional referral center for trauma, neurologic emergency, thoracic surgery, and plastic surgery patients. On average, there are about 8,000 ED visits and 1,200 trauma patients brought to the hospital per month. The study was approved by the hospital's Human Experiment and Ethics Committee. Patient data were collected prospectively and entered into our Institutional Trauma Registry databank. Patients who sustained motorcycle-related accidents and were brought to our ED from January $1^{\text {st }} 2007$ to December $31^{\text {st }} 2011$ were enrolled into the survey. We only included those patients with motorcycle-related injury brought by EMTs because the procedures of pre-hospital rescore are more standardized. During the study period, patients who were unconscious, died at the ED, had incomplete data, or were resuscitated after being hemodynamically unstable, were excluded.

Data Collection

For each enrolled patient, a standardized trauma sheet was used to record the patients' demographics, clinical data, trauma mechanism, imaging results, and neurological exams. All enrolled patients received a neurological examination after arrival and once again before leaving the ED by the same senior trauma surgeon (the study coordinator). Variables collected at our Institutional Trauma Registry databank include age, gender, mechanism of injury, the number and portion of spinal columns injured, Injury Severity Score (calculated using version AIS-90), Glasgow Coma Scale (GCS), neurological exams, and need for operation. Patients who were not examined by the trauma surgeons, had associated injuries that needed immediate medical intervention without a complete neurological exam, a GCS less than 15, left against medical advice, or had missing data, were excluded (figure 1). A trained chart abstractor, who is a registered trauma nurse, computed the data and recorded the trauma registration. With a standardized form to guide data collection, data were collected in a prospective fashion. When data were found to be conflicting, ambiguous, missing, or unknown, then a senior trauma surgeon was placed in charge of periodic weekly meetings with the study coordinators to ensure uniform handling of the data, resolve disputes, and review coding rules. The performance of the chart abstractor was monitored by the senior surgeon working in the same office/room. The abstractor was blinded to the study and the group that the patients were assigned to.

Methods of measurement

Patients were managed under the protocols of ATLS. They received primary and secondary examination to exclude critical injuries. Upon arrival, they were transferred from the board, used by the EMTs at the scene of the accident, to a hospital bed. The extrication board, when used, was removed during the logroll procedure in order for the physician to examine the patient's posterior surface in the initial assessment phase. Patients suspected of having a spinal injury were examined by trauma surgeons involved in the study using a more detailed sensory and motor function test for lesion detection, according to the Standard Neurological Classification of Spinal Cord Injury. The Standard Neurological Classification of Spinal Cord Injury test includes a motor exam of ten key muscles with a 6 point scale $(0-5)$ and 28 sensory tests (light touch and pin pain) with a 3 point scale (0-2). The neurological examinations of the enrolled patients were measured by the same trauma surgeon upon arrival and before leaving the ED. 
Table 1. Neurologic exam according to the Standard Neurological Classification of Spinal Cord Injury.

\begin{tabular}{llll}
\hline Sensory (score) & Arrival & Before leaving & $\mathrm{p}$ \\
\hline Light touch & & & \\
$\quad$ Right & $46.7 \pm 26.4$ & $43.7 \pm 25.2$ & 0.552 \\
Left & $45.7 \pm 27.3$ & $44.1 \pm 24.6$ & 0.429 \\
& & & \\
Pin prick & $44.2 \pm 26.8$ & $43.6 \pm 25.5$ & 0.267 \\
Right & $43.9 \pm 25.3$ & $42.5 \pm 27.2$ & 0.356 \\
Left & & & \\
& & & 0.114 \\
Muscle motor exam & $39.5 \pm 12.3$ & $38.5 \pm 14.2$ & 0.159 \\
Right & $38.2 \pm 16.9$ & $37.6 \pm 17.4$ & \\
Left & & & 0.432 \\
Total $(\mathrm{n}=91)$ & $252.3 \pm 82.1$ & $248.9 \pm 87.8$ & \\
\hline
\end{tabular}

$\mathrm{p}$, Repeated measures analysis.

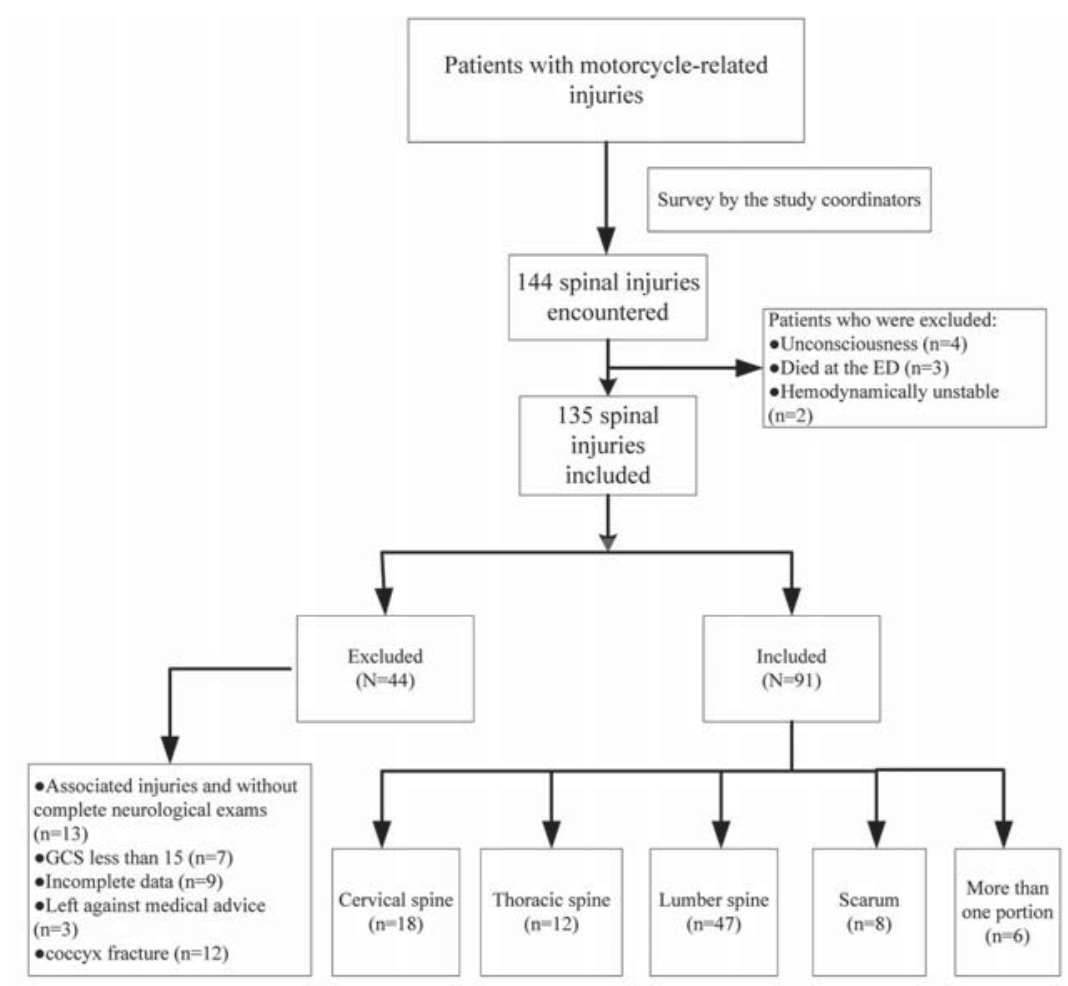

ED, emergency department; GCS, Glasgow Coma Scale.

Figure 1. Summary of included and excluded patients. Incomplete data includes any patients who had missing information or unavailable neurologic exam status.

A neurosurgeon was consulted for further management if spinal injury was detected. Likewise, surgical interventi- ted tomography (CT), magnetic resonance imaging (MRI) or X-ray reports, as interpreted by radiologists. The definition of spinal injury includes any stable or unstable lesion, such as fracture and hemorrhage found on the final radiological reports involving the nerves, spinal column, cord, or both. Soft tissue injuries, such as tearing of ligaments or muscles, were also included.

Statistical Analysis

Data analysis was performed using the SPSS statistical package (SPSS Version 15.0, SPSS, Chicago, IL). All continuous variables, including age, neurological exam scores after arrival and before leaving, and hospital length of stay, were reported as a mean $( \pm S D)$. The repeated measures analysis (2-tailed) was used to examine whether or not there were any changes between the neurological tests and immobilization. The distribution of patient characteristics and survey results were summarized using simple descriptive statistics. A p value of 0.05 or less was considered statistically significant.

\section{Results}

During the study period, 144 patients had blunt spinal injuries and were surveyed by the trauma surgeons involved in this study. After excluding patients with unconsciousness $(n=4)$, those who died at the ED $(n=3)$, or were hemodynamically unstable $(n=2), 13$ patients without complete neurological exams, 12 patients with a coccyx fracture and 7 patients with a GCS score less than 15 were also excluded. Nine patients had incomplete data and 3 patients that left the hospital, despite medical advice to the contrary, were also excluded. After these patients were excluded (36.8\%; 53/144), there were $91 / 144(63.2 \%)$ patients in total with 19.8\% (18/91) cervical, 13.2\% (12/91) thoracic, 51.6\% (47/91) lumbar, 8.8\% (8/91) sacral, and 6.6\% (6/91) with more than 2 areas with spinal injuries enrolled. Their mean age was $45.44 \pm 18.12$ years with $38.4 \%(35 / 91)$ male and $61.6 \%$ (56/91) female patients. Upon arrival, patients were put on a hospital gurney (figure 1). 


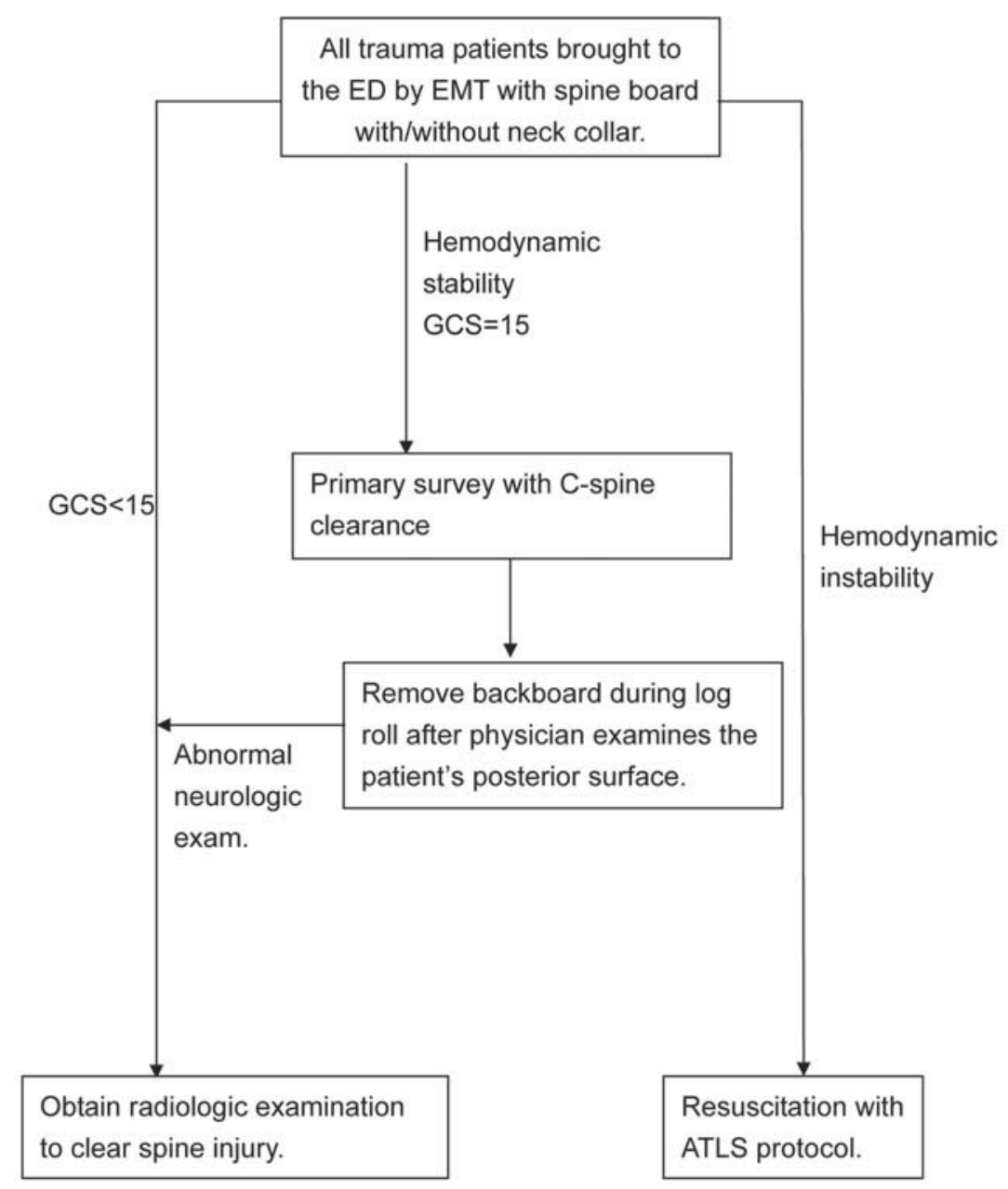

ATLS, advanced trauma life support; ED, emergency department; EMT, emergency medical technicians; GCS, Glasgow coma scale.

Figure 2. Institutional protocol for spine board removal.

Ninety-two percent (84/91) of patients were responsible for driving the scooters themselves while the others were just passengers. Eighty-two percent (75/91) wore helmets, 6.6\% (6/91) did not wear any protective device whatsoever and in $11.0 \%$ (10/91) of patients, it was unclear whether they were wearing a helmet or not. The associated injuries included 20.9\% (19/91) head and neck injuries, $11.0 \%$ (10/91) facial injuries, $10.0 \%$ (9/91) chest injuries, $13.19 \%$ (12/91) abdominal injuries, and 98.9\% (90/91) injuries to the extremities. The average time from the ED to the hospital ward was $11.2 \pm 4.8$ hours. The average length of stay in the hospital was $3.5 \pm 5.1$ days. Only four patients had cervical spine injuries requiring surgical fixation and were admitted to the intensive care unit. Another five patients with thoracic and lumber spinal injuries received emergency decompression and fixation.

The neurologic exams, including sensory (light touch and pin prick) and motor tests, after arrival and before leaving the ED, tested by Repeated Measures analysis, showed no significant differences $(p=0.432)$ (table 1$)$.

\section{Discussion}

Although an extrication board can be used to assist in the transport of a patient, those who have sustained a blunt spinal injury with clear consciousne- ss can be placed on a gurney without compromising the patient's condition after removal of the extrication board at the hospital.

According to the training of EMTs, at the scene of an accident, if spinal injury is suspected, a spinal board with a neck collar should be applied to prevent further spinal damage. However, there is still controversy pertaining to the overuse of spinal boards and neck collars in the prevention of spinal injury. For neck collar use, according to the Eastern Association for the Surgery of Trauma (EAST) guidelines (2009) from the USA, one suggestion is to remove the neck collar as soon as possible without the need for further imaging in awake, alert trauma patients, if the cervical spine is clear. Restraint with a neck collar and spinal board has adverse effects on the body such as respiratory distress, pressure sores, discomfort, and the potential for choking on vomit etc. (3,9-11) Therefore, after arriving at the hospital, removal of the spinal board and neck collar is suggested after evaluation by a physician, if the patient has been cleared of spinal injury. (7) Although a neck collar may be applied until imaging clears the possibility of cervical injury, under the discretion of different physicians, most of the time the spinal board should be removed with the log roll or lift and slide maneuver $(12,13)$ and the patient should be put on a hospital gurney with a sponge, if no obvious tenderness or pain over the patient's back is readily apparent. The newly developed, soft-layered, long spine board should be considered to reduce tissue-interface pressure and increased comfort of patients. (14) Although a support-surface with a semi-soft overlay mattress or a vacuum mattress could be used and is better than a spine board, (15) in our study, regardless of whether the spinal board was used or not, we did not find any obvious deterioration in the results of the neurological exams after moving the patient to a standard bed. Putting patients in a bed will not lead to any compromise of neurological function. During the waiting period and transportation to the ED, it is not necessary to use the spinal board. 
In our study, some patients without abnormal neurological exams were brought to our hospital with a spinal board and neck collar. Although not all spinal injuries show obvious signs of neurological deficit at the scene of the accident, according to a previous study, a spinal board should be used with caution and according to the situation, rather than simply applying them to everyone. (10) Recently, owing to the rigid board not conforming to the shape of a patient's back, there have been many studies providing support that this method is inferior to other methods of immobilization, such as a vacuum or other mattress. $(16,17)$ Immobilization of patients on a spine board with an interposed air mattress or increasing the amount of padding have also been proposed. $(10,18)$ However, patients should finally be put on a hospital bed and the supporting mattress removed. The use of spinal boards and neck collars should be limited to patients that are unconscious, have neurological deficits, and have no vital signs at the scene, rather than someone without any neurological exam abnormalities.

In this study, all patients had motorcycle-related injuries. Motorcycle riding is a recreational activity in most Western countries, but it is a common mode of transportation in some European and Asian countries. In Taiwan, the density of motorcycles is 1.4 motorcycles per household on average, the highest in the world, resulting in a stepwise increase of motorcycle-related accidents in the past few years. Most of the patients who sustained light-weight motorcyclerelated accidents did not have cervical spinal injuries. (10) Motorcycle-related injuries in an urban area characteristically occur at lower speeds (less than $60 \mathrm{~km} /$ hour). The speed limit and traffic congestion in the city slow down the riding speed, and people who do get hurt, can be brought to a hospital for proper care, resulting in a decrease in the severity of injuries during and after impaction. In this study, we found that although patients may have a higher injury severity score (ISS) due to a relati- vely unprotected body, their extremities are more prone to injury than the spine. More female than male patients were included, because males were more prone to sustaining severe injuries and therefore excluded, presumably because of driving at higher speeds or being more involved in risk-taking. The lumbar spine was found to be the most injured region followed by the thoracic spine and sacrum respectively. Coccyx injury is unusual in this type of study and was excluded; however, patients with sacrum injury might have pain radiating to the lower back and therefore could not be distinguished from patients with lumbar injury. The rate of cervical spine injury was the lowest.

In our study, only 9 patients were found to have an unstable spinal injury and therefore only these 9 were at risk of any sort of neurological deterioration during their ED stay. To mix the results from these 9 with the 68 patients that did not have an unstable spinal injury makes it very difficult to detect any significant neurological changes, and there would be an argument for presenting the results of the 9 patients alone, though the numbers would then be too small to draw any useful conclusions.

Limitations

There are several limitations to our study including the potential for observer bias, given that the trauma surgeons were not blinded to the aims of the study. Nevertheless, two senior, experienced trauma surgeons recruited the study participants and were responsible for data collection, which in turn, increased the accuracy of the study. Additionally, a standard data collection tool was used for demographic data collection before the patient left the ED, and the data were completed consistently according to protocol. Further limitations include the use of a single medical center and the potential inaccuracies of history-reporting from patients, whose quality of recall about the mechanism of injury is often unable to be corroborated. Cervical spine injury, likely to be insensitive to backboard support, may coincide with other spinal injuries and requires that the patient be placed on an extrication board. Therefore, we included this in our analysis. Furthermore, the neurological exams between the different surgeons may perhaps have varied slightly. However, patients were examined by the same physician, thus the neurological exams done before and after should have been consistent. Although in some cases, if examinations of patients were performed by the same person within a short time frame thereby possibly introducing a substantial risk of bias, the average time between the examinations was $11.2 \pm 4.8$ hours. In the study, we did not follow-up with a neurological exam before the patient was discharged. Therefore, the outcome of the patient's neurological status is unknown. However, some patients may have received some kind of treatment after admission to improve their neurological function. In the pre-hospital phase, it is unclear which patients were placed on backboards by EMT's, who needed complex rescue support, and who suffered severe transient hypotension, which may affect neurological outcome. For this reason, we excluded those with a relatively unstable hemodynamic status requiring resuscitation. Thus, further study is warranted for patients with these complications. Finally, our study is a medical center study, and therefore, it is possible that the results may not apply to other institutions. However, given that the institution is a major referral center for trauma from metropolitan and regional trauma services, the participants were most likely a strong representation of patients presenting to metropolitan and regional centers.

\section{Conclusion}

In this study, neurological examinations of spinal injury patients did not change significantly between the time of arrival and when leaving the ED without the use of an extrication board. If excessive movement of the spine is avoided with the log roll or sliding board method during examination and transportation, the patient can be safely placed on a hospital gurney without causing further damage. 


\section{ACKNOWLEDGMENT}

We would like to thank Shing-Ghi Lin for his assistance with the table and figure preparation of this manuscript and Dr. Ching-Ying Wu and Morgan Harloff for assistance with manuscript submission and editing. The first two authors made equal contributions to this work and are equally considered to be first author.

\section{REFERENCES}

1. De Lorenzo RA. A review of spinal immobilization techniques. J Emerg Med 1996 Sep-Oct;14(5):603-13.

2. Kosashvili Y, Backstein D, Ziv YB, Safir O, Blumenfeld A, Mirovsky Y. A biomechanical comparison between the thoracolumbosacral surface contact area (SCA) of a standard backboard with other rigid immobilization surfaces. J Trauma 2009 Jan;66(1):191-4.

3. Burton JH, Dunn MG, Harmon NR, Hermanson TA, Bradshaw JR. A statewide, prehospital emergency medical service selective patient spine immobilization protocol. J Trauma 2006 Jul;61(1):161-7.

4. Edlich RF, Mason SS, Vissers RJ, Gubler KD, Thacker JG, Pharr P, et al. Revolutionary advances in enhancing patient comfort on patients transported on a backboard. Am J Emerg Med 2011 Feb;29(2):181-6.

5. Chan D, Goldberg RM, Mason J, Chan L. Backboard versus mattress splint immobilization: a comparison of symptoms generated. J Emerg Med 1996 May-Jun;14(3):293-8.

6. Lerner EB, Moscati R. Duration of patient immobilization in the ED. Am J Emerg Med 2000 Jan;18(1):28-30.

7. Hauswald M, Braude D. Spinal immobilization in trauma patients: is it really necessary? Curr Opin Crit Care 2002 Dec;8(6):566-70.

8. Hauswald M, Ong G, Tandberg D, Omar Z. Out-of-hospital Spinal Immobilization: Its Effect on Neurologic Injury. Acad Emerg Med 1998:5(3):214-9.

9. Hauswald M, McNally T. Confusing extrication with immobilization: the inappropriate use of hard spine boards for interhospital transfers. Air Med J 2000 Oct-Dec;19(4):126-7.

10. Cordell WH, Hollingsworth JC, Olinger ML, Stroman SJ, Nelson DR. Pain and tissue-interface pressures during spine-board immobilization. Ann Emerg Med 1995 Jul;26(1):31-6.

11. Kwan I, Bunn F. Effects of prehospital spinal immobilization: a systematic review of randomized trials on healthy subjects. Prehosp Disaster Med 2005 Jan-Feb;20(1):47-53.

12. Horodyski M, Conrad BP, Del Rossi G, DiPaola CP, Rechtine GR 2nd. Removing a patient from the spine board: is the lift and slide safer than the log roll? J Trauma 2011 May; 70(5):1282-5; discussion 5.

13. Del Rossi G, Horodyski M, Heffernan TP, Powers ME, Siders R, Brunt D, et al. Spine-board transfer techniques and the unstable cervical spine. Spine (Phila Pa 1976) 2004 Apr 1;29(7):E134-8.

14. Hemmes B, Poeze M, Brink PR. Reduced tissue-interface pressure and increased comfort on a newly developed soft-layered long spineboard. J Trauma 2010 Mar;68(3):593-8.

15. Keller BP, Lubbert PH, Keller E, Leenen LP. Tissue-interface pressures on three different support-surfaces for trauma patients. Injury 2005 Aug;36(8):946-8.

16. Luscombe MD, Williams JL. Comparison of a long spinal board and vacuum mattress for spinal immobilisation. Emerg Med J 2003 Sep;20(5):476-8.

17. Johnson DR, Hauswald M, Stockhoff C. Comparison of a vacuum splint device to a rigid backboard for spinal immobilization. Am J Emerg Med $1996 \mathrm{Jul} ; 14(4): 369-72$

18. Hauswald M, Hsu M, Stockoff C. Maximizing comfort and minimizing ischemia: a comparison of four methods of spinal immobilization. Prehosp Emerg Care 2000 Jul-Sep;4(3):250-2. 